\title{
OSCILLANDO TRA METODO JAHR̄̄ E KHĀFī NELLA VALLE DEL FERGANA
}

\section{Giovanni De Zorzi (Università "Ca' Foscari" di Venezia)}




Riassunto: Questo articolo prende in esame i metodi di realizzazione spirituale di alcuni dervisci detti naqsbandī jahrī incontrati nella valle del Fergana (Uzbekistan), i quali impiegavano sia lo zikr khäf ("silente, interiore") che quello jahrī ("sonoro, manifesto"). Più in generale, in Asia centrale il modo di esecuzione dello zikr connota e distingue due scuole dette, significativamente, khäfiyya e jahrîyya, ognuna delle quali prevede, oltre allo zikr, una serie di pratiche che le sono tipiche e che vengono qui isolate, descritte e analizzate partendo dalle informazioni raccolte durante una ricerca sul campo svolta secondo i metodi dell'etnomusicologia, dell'antropologia e dell'orientalismo. In particolare, vengono trascritti e analizzati alcuni peculiari tipi di $z i k r$, sia $k h \bar{a} f \bar{\imath}$ che $j a h r \overline{\text {, e }}$ un inedito cerimoniale detto raqs-i sama $\bar{a}^{\varsigma}$ (raqs-u samo) per il quale si ipotizza, in base a diversi elementi, un ricollegamento con i dervisci naqshbandī a âa $\bar{q} \bar{\imath}$, attivi sin dal XVIII secolo sui territori dell'attuale Xinjiang cinese. L'articolo termina, infine, con alcune osservazioni dei dervisci intervistati sui valori funzionali di alcuni tipi di $z i k r$.

Parole chiave: $d h i k r$; zikr; jahrī; khāfí; naqsbandīyya; yasavīyya; Storia del sufismo (tasawreuf) in Asia centrale. $\therefore$

Abstract: This article takes into exam the methods of spiritual realisation of some dervishes called naqsbandī jahri I've met in the Fergana valley (Uzbekistan), who used both the zikr khāfi ("silent, inner") and the zikr jahri ("loud, manifest"). More generally, in Central Asia the way of performing the $z i k r$ connotes and distinguishes two schools, significantly called khäfizya and jahriyya, each of which includes, in addition to their specific $z i k r$, a series of practices that are typical of the same school. Such practices are isolated, described and analysed here on the basis of information gathered during fieldwork carried out according to the methods of ethnomusicology, anthropology and orientalism. In particular, some peculiar types of $z i k r$ are transcribed and analysed, as well as an unpublished ceremonial called raqs-i sama $\bar{a}^{\sigma}$ (raqs-u samo) for which, on the basis of various elements, I suppose a connection with the naqshband $\bar{\imath} \bar{a} \bar{a} q \bar{\imath}$ dervishes, who lived since the 18th century in the territories of present-day Chinese Xinjiang. The article ends with some observations of the interviewed dervishes on the functional values of some types of zikr.

Keywords: dhikr; zikr; jahrī; khāfī; naqshbandīyya; yasavīyya; History of Sufism (tasawreuf) in Central Asia.

Ad Alberto e Paolo Ventura con profonda gratitudine 
Queste pagine nascono da una serie di incontri che ebbi nel 2003 nella parte uzbeca della valle del Fergana con dei dervisci che lo storico del sufismo Thierry Zarcone definisce naqshbandījahri. La sede centrale della confraternita era la dimora privata di un anziano Adil Khan Qāri, nato nel 1920, riconosciuto esponente di una catena di discendenza spirituale che risaliva ad un lontano passato e che non si era interrotta durante la repressione russo-sovietica ${ }^{1}$. Quando lo visitai, il maestro era agli arresti domiciliari per aver definito i rappresentanti del governo di allora come dei "corrotti". La sua casa era sotto sorveglianza della polizia, ma era il punto di riferimento per dervisci che provenivano da ogni parte del Fergana e dell'Uzbekistan, soprattutto in occasione dello zikr del giovedì sera. Una delle particolarità del

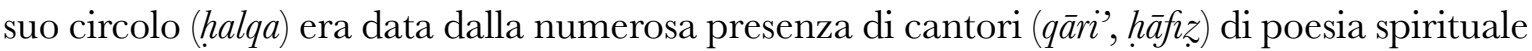
tradizionale, com'era lui stesso. Le prossime righe prendono lo spunto da incontri avuti nella sua casa di Andijān così come con altri esponenti della sua scuola in altri luoghi del Fergana e a Safìd Bulān, nel Kyrgyzstan. ${ }^{2}$

La definizione naqshbandī-jahrī sintetizza la commistione dei metodi di realizzazione spirituale adottati nella corrente di Adil Khān, i quali, di là da certi rigidi e forse comodi schematismi, provenivano dalla scuola khāfì così come da quella jahrī. Converrà ricordare che il modo di esecuzione del zikr fu nel tempo la principale linea di demarcazione all'interno del mondo sufi centroasiatico, diviso in due correnti principali: chi praticava il zikr jahri ("vocale, sonoro, manifesto") e chi praticava il zikr khāfí ("nascosto, interiore, silente"). Nei secoli il termine si spostò dal mero modo di esecuzione dello zikr e divenne gradualmente sinonimo di un sistema complessivo, così che si distinsero due principali correnti dette jahrīyya e khäfīyya (anche khufiyya). Si soleva poi ricondurre, davvero molto schematicamente, le due vie e i due approcci a due gruppi etnici: la jahrīyya alle genti nomadi di lingua turca e ai loro maestri, i cosiddetti mahashaykh-i-türk (su tutti Aḥmad Yasavī, m. 1166), la khāfīyya alle genti stanziali di lingua persiana e ai loro maestri, i khwäjagān. Dagli insegnamenti dei mahashaykh-i-türk e dei khwäjagān sarebbero fiorite due vie: la tariqqa yasavīyya e la tarīqa-i khwäjagān, che in seguito prese il nome di tarīqa-i naqshbandiyya dal suo eponimo Bahā ud-Dīn Naqshband (m. 1389) ${ }^{3}$.

1 Thierry Zarcone, "Bridging the Gap between pre-Soviet and post-Soviet Sufism in Ferghana Valley (Uzbekistan): The Naqshbandi Order between Tradition and Innovation" in Popular Movements and Democratization in the Islamic World, edited by Masatoshi Kisaichi, New Horizons in Islamic Studies, New York, Routledge, 2006: 46-47.

2 Da allora nel corso degli anni ho affrontato la mia esperienza sotto varie angolature, dall'iniziale tesi di dottorato del 2006, ricca di allegati audio e video, ad una serie di articoli per i quali rinvio alla bibliografia. La mia ricerca iniziava dal lavoro di Alberto Ventura ma "fisicamente" partiva dalla casa romana di Paolo Ventura: ringrazio entrambi ancora una volta dedicando loro queste pagine.

3 Un quadro complessivo tra jahrīyya e khāfìyya in Bakhtiyar Babadjanov, Sanjar Ghūlamov, "Ritual practice of Sufi communities in Mavara'annahr (18th-19th centuries)" in Central Asia on Display. Proceedings of the VII European Society for Central Asian Studies Conference, a cura di Gabriele Rasuly-Paleczek e 
Inserita nel panorama di cui sopra, la corrente dei dervisci naqshbandījahri incontrati nel Fergana potrebbe dunque sembrare un po' eccentrica rispetto alla corrente centrale

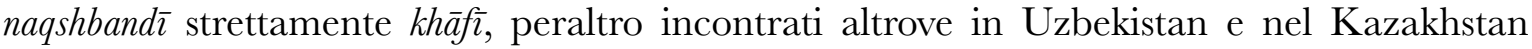
meridionale sotto la guida del carismatico Ibrāhim (o Ibrāhimjān) sheykh. I dervisci del Fergana praticavano zikr khäfi e zikr jahrī, talora operando delle commistioni tra i due: essi sostenevano, ad esempio, che un determinato zikr jahrī, come ad esempio quelli del tipo yek, do, se, chahār zarb, che vedremo meglio in seguito, poteva essere eseguito su/con uno dei particolari centri sottili (latāâtif) dell'essere umano, tipici della mappatura khäfì zikr yek zarb su laț̃̄a qalb, zikr do zarb su laț̄fa rūh, eccetera. Soprattutto, essi praticavano un particolare rituale di raqs-i sama $\bar{a}^{‘}$ ("moti che nascono dall'ascolto") che costituisce un caso piuttosto raro nel vasto mondo naqshbandī ma che, a mio avviso, può essere ricollegato a pratiche analoghe tra naqshbandī del Turkestan orientale, l'attuale Xinjiang.

Nelle prossime pagine inizierò con il mettere a fuoco alcune componenti del metodo naqshbandī jahrī; passerò, poi, a isolare alcuni singoli e caratteristici tratti di zikr khäfi e jahrī, per concentrarmi quindi sul rituale di raqs-i sama $\bar{a}^{\complement} \mathrm{e}$ i suoi possibili collegamenti con il mondo sufi degli uiguri, nel Turkestan orientale. In conclusione, seguendo le parole dei dervisci incontrati, cercherò di delineare una complessiva "funzionalità" del loro metodo.

\section{ELEMENTI DEL METODO JAHR $\bar{I}$}

Tra le pratiche tipiche del metodo jahri svetta, naturalmente, il particolarissimo tipo di $z i k r$ che in area centroasiatica si presenta come uno zikr eseguito perlopiù collettivamente, dal grande volume sonoro, con un'emissione vocale spesso "lacerata", prossima al canto difonico di stile kargirää della vicina Mongolia, e con una forte attenzione alla scansione ritmica. Ogni singolo tipo di $z i k r$ ha poi una sua storia particolare, una sua genesi e viene messo in relazione con una data figura spirituale, ad esempio Adamo, il Profeta Zaccaria, Ạ̣mad Yasavī o altri maestri. Nella riunione collettiva di un circolo (halqa) lo zikr si alterna, o si combina, con il canto di composizioni poetiche nate da poeti sufi di area centroasiatica.

Julia Katschnig, LIT Verlag, Wien, 2004, Volume 1: 297-308. Sulla Yasavīyya e su alcuni suoi specifici zikr jahrī: Bakhtiyar Babadjanov, "Une nouvelle Source sur les rituels de la tarīqa Yasavìyya. Le risala-i dhikr-i sultān al-'arifinn", in fournal d'Histoire du Soufisme, n. 3, 2002, Maisonneuve, Paris: 223-228. Sul zikr khăfì e sulla sua progressiva adozione: Hamid Algar, "A brief history of the Naqshbandī Order" in Marc Gaborieau, Alexandre Popovic e Thierry Zarcone (a cura di), Naqshbandis. Cheminements et situation actuelle d'un ordre mystique musulman, Işin, Istanbul, 1990: 3-44. In epoca russo-sovietica, la pratica dell'uno o dell'altro tipo di zikr ebbe un riflesso politico: i dervisci che seguivano la via khā $\bar{\imath}$, meno "udibili" e visibili nei loro cerimoniali, sopravvissero meglio alla repressione, si veda: Alexandre Benningsen, Chantal Remercier Quelquejay, L'Islam parallelo. Le confraternite musulmane in Unione Sovietica, Marietti, Genova, 1990. (ed. or. Le soufi et le commissaire, Editions du Seuil, Paris, 1986). 
Questo ci porta ad un secondo elemento tipico del metodo jahri in area centroasiatica che consiste in quelle particolari figure di cantori professionisti, detti hāfiz, oppure qāri', che cantano/declamano composizioni di poeti sufi durante gli zikr. Questo avviene in due modalità principali: il loro canto può elevarsi sull'ostinato costituito dallo zikr stesso, oppure in solo, mentre lo zikr tace. Quest'ultima modalità ha il duplice scopo di placare gli animi, consentendo un breve riposo agli zākir ("colui che pratica lo zikr") così come di riaccenderne, in un secondo momento, il fuoco.

Uscendo dal bruciante contesto dei circoli (halqa) di zikr, gli hă $f i z$ possono cantare, come ho visto accadere, in incontri di altre confraternite oppure in occasioni e cerimonie legate alla vita comunitaria quali nascite, circoncisioni, matrimoni e morti, detti sempre e comunque toy ${ }^{4}$. Talvolta alcuni $h \bar{a} f \imath z$ sono, o sono stati, dei cantanti professionisti: un caso esemplare fu quello dei due fratelli Akmāl-khān e Bābā-khān Subhanov (anche Sofikhanov), formatisi come hāąiz nel circolo di zikr condotto dal padre. Noti e amati per il loro repertorio di canti d'argomento spirituale, essi dovettero lasciare la natia Yasi (oggi Turkistan, nell'attuale Kazakhstan) per sfuggire al bando culturale delle autorità rifugiandosi a Tashkent, dove registrarono dei brani per la radio nazionale che si possono ora ascoltare in Ouzbékistan, Grandes voix du passé (19401965), a cura di Jean During e Abdurahim Hamidov (Ocora-Radio France, Paris, 1999, CD C 560142: 1-4) $)^{5}$.

Il corrispettivo femminile degli hă $f \imath z$ sono le otin (talora otin o $)$, anch'esse interpreti di testi spirituali: come le loro controparti maschili, le otin cantano i loro repertori durante le sedute femminili di zikr, oppure durante riunioni dette mushkelgusha ("risoluzione di problemi"), nelle quali testi e formule spirituali hanno una precisa funzione terapeutica ${ }^{6}$. Il concetto di

4 Il termine toy indica la festa per un momento di passaggio nella vita di un singolo o di una comunità. Ovviamente l'atmosfera cambia secondo i contesti ma si può dire che, a prescindere dalle occasioni, la caratteristica comune sia quella di invitare, ospitare e sfamare molte persone. Nel toy per un matrimonio esplode la musica, la danza e le famiglie degli sposi invitano i parenti allargati e tutto il vicinato a feste alle quali partecipano centinaia di persone.

5 I due fratelli e il loro circolo di zikr a Yasi sono ricordati con affetto e nostalgia dal grande solista uzbeko Turgun Alimatov in una sua lunga intervista concessa all'etnomusicologo Theodore Levin nel suo fondamentale Theodore Levin, The hundred thousand Fools of God. Musical travels in Central Asia (and Queens, New York), Indiana University Press, Bloomington, 1996: 62-65. Va anche citato il caso del cantante, musicista e compositore Arif Khatamov e la sua ricostruzione dell'ambiente degli zikr ai quali partecipò a Yasi ancora in Levin, 1996: 75-76.

6 Eckhard Neubauer - Veronica Doubleday, "Islamic Religious Music. 3 Dhikr and sufi ritual" in The New Grove Dictionary of Music and Musicians, Macmillan Publishers, 2001, Volume 12: 605. Sulle otin Habiba Fathi, "Le pouvoir des otin, institutrices coraniques, dans l'Ouzbékistan indépendant", in Boukhara la noble. Cahiers d'Asie Centrale, 5-6, Édisud, Tachkent-Aix en Provence, 1998: 313-331. Razia Sultanova, "En soulevant le voile: les chants des femmes dans les cultures traditionnelles turco- 
guarigione dipende ancora una volta dal potere insito in alcune formule sacre come quelle dello zikr, composte di nomi divini o formule coraniche, le quali, essendo esteriorizzate e

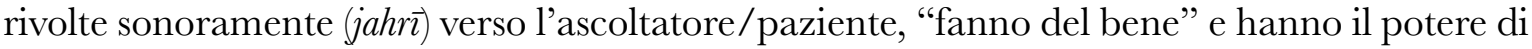
guarire corpo ed anima. Nei rituali da me osservati, soprattutto in quelli dettijahr e damsolish, l'officiante somministrava al paziente dei simbolici colpi di frusta (kancı) che scacciavano demoni e spiriti. La vicinanza con pratiche di guarigione sciamanica è piuttosto evidente e, complessivamente, queste pratiche di guarigione sono state definite da alcuni come "sciamanesimo islamizzato" oppure "Islam sciamanizzato" 7.

Durante uno zikr jahrī, infine, può aversi il cosiddetto raqs-i sam $\bar{a}$, termine traducibile letteralmente con "moti e ascolto", e, più liberamente con "moti generati dall'ascolto". Dall'ascolto dei versi cantati dagli hăfiz, o dalle otin, sull'ostinato dello zikr, possono sorgere nei presenti particolari movimenti fisici detti raqs ("moti, movimenti"), che possono essere più $\mathrm{o}$ meno composti, più o meno prefissati e sui quali mi dedicherò a parte.

Alle pratiche elencate può essere aggiunto il periodo di quaranta (cihil) giorni di assoluto ritiro dal mondo (tarq-i dünya) detto chilla, che molti dervisci jahrī, soprattutto quelli yasavi, compiono in onore e ad imitazione del loro maestro.

\section{CONGENTRANDOSI SUGLI ZIKR}

Nelle prossime righe sarà di riferimento un passaggio dell'etnomusicologo Jean During che isola sette tipologie di $z i k r^{8}$ :

1. "ricordo" senza supporto verbale;

2. zikr silente;

3. zikr verbale;

4. zikr verbale che fa da ostinato ad una melodia;

5. melodia che include una formula di zikr (a volte in forma di "ritornello" o refrain);

6. melodia che diviene progressivamente uno zikr;

7. melodia il cui ritmo suggerisce la presenza dello zikr (verbale o silente).

phones", in Cahiers des Musiques Traditionnelles, Genève, 2005, n. 18: 11-38.

7 Si veda soprattutto Patrick Garrone, Chamanisme et Islam en Asie Centrale, Maisonneuve, Paris, 2000; Anne-Marie Vuillemenot, "Quand un bakhshı kazakh invoque Allāh " in "Journal d'Histoire du Soufisme”, n. 4, Maisonneuve, Paris, 2004: 131-141.

8 Jean During, Musica ed Estasi. L'ascolto mistico nella tradizione sufi. Traduzione e cura di Giovanni De Zorzi, Roma, SquiLibri: 83. (ed. or. Musique et Extase. L'audition mystique dans la tradition soufie, Paris, Albin Michel, 1988). 
Ebbene, i tipi di zikr da me incontrati nelle comunità del Fergana rientrano certamente nelle tipologie che vanno dal punto due al punto sei, ma tra esse ha una posizione preponderante la tipologia al punto quattro, quando su di un ostinato costituito dallo zikr dei presenti stessi si eleva il canto di un solista, oppure quando il canto diviene collettivo.

Come si diceva, la scuola di Adil Khān praticava indifferentemente zikr khāfi e jahrī; quanto al primo, più difficilmente documentabile, prendo spunto da un'intervista avuta con un gentilissimo testimone, haj̋ A. M. di K., intervistato il 13 aprile 2003, secondo il quale il metodo praticato nella loro via prevedeva:

Tauba, ("pentimento"): si doveva pronunciare intimamente khāfi (in uzbek, khufi) la formula: Astaghfirullāh;

Pronunciare khufi 300 volte bismillah ar rahman ir rahim;

Pronunciare khufi 100 volte la formula: La nest, la, ha illa Llah, Hu, Maqsudi, illa Lahi juzi zat-i pak ("Non v'è, no, altro dio se non Iddio, Lui, colui al quale si tende, possessore dei Nomi divini");

Zikr khufi di Abu Bakr Siddiq: si deve pronunciare ad alta voce il nome divino Allāh "con la lingua", una sola volta e poi continuare a pronunciare il nome in silenzio (khufi) sul tesbih ("rosario") per 100 volte senza più prendere fiato. "Noi normali riusciamo a farlo per 33 volte", mi diceva, "ma l'importante è il respiro, non il numero: si può eventualmente, ogni tanto, 'rinfrescare' il respiro dal naso ma mai espirare."

Passo ora a trascrivere e contestualizzare alcuni degli dhikr jahrī che ho potuto ascoltare tra varie comunità; va notato che i dervisci incontrati ritenevano la trascrizione, così come le registrazioni audio e video, importanti mezzi per preservare e trasmettere la loro tradizione.

\section{ANTALHO}

Inizio con un caso un po' particolare, quello dell'Antalho: questo può essere definito un "inno sacro" (ilāhi) che inserisco qui soprattutto perché era presente nelle cerimonie di tutti i dervisci incontrati durante la mia ricerca: tra i vari centri sufi del Fergana, così come a Safìd Bulān, nel Kyrgyzstan, oppure a Turkistan ed Almaty nel Kazakhstan meridionale, a Bāysun, nel Surkhandārya. Ovunque si fosse, tutti i cerimoniali si aprivano, o inserivano immediatamente nella prima fase, questo inno che sostiene:

Anta l-Hādi, Anta l-Haqq,

Laysa al-Hādi illā Hū, 
Hasbī Rabbī Jalla Llāh, mā fì qalbī ghayru Llāh,

Nūru Muhammad șalla Llāh, lā ilāha illā Llāh $h^{9}$

"Tu sei la Guida, Tu sei il Vero, non v'è altra Guida se non Lui,

Faccio conto sul Signore, che sia celebrato! (Non c'è) nulla nel mio cuore che non sia Iddio,

Luce di Muhammad, Iddio preghi su di lui, non v'è altro dio se non Iddio".

Secondo l'etnomusicologa Razia Sultanova, l'inno, noto come "Antalho", sarebbe una formula melodica di zikr originaria della Yasavĩyya diffusasi in seguito in tutto il mondo islamico. In questa prospettiva, l'Antalho è di per sé una forma di zikr che rientra, così, nello schema riassuntivo proposto dell'etnomusicologo Jean During, nel quinto punto, in quanto: "melodia che include una formula di $z i k r$ in forma di 'ritornello' o refrain", laddove l'evidente ritornello è lā ilāha illa Llāh. Ancora secondo la Sultanova, in senso più generale, anche brani puramente strumentali sarebbero influenzati dalla formula ritmica intrinseca nell'attestazione di fede (shahāda) lā ilāha illā Lläh. Tale formula ritmica viene detta chāhär (uzbeco chôr) zarb ("quattro tempi, colpi"). Ecco la trascrizione dell'Antalho, si noti, però, come la trascrizione sia stata portata all'ottava più alta per una maggiore chiarezza visiva

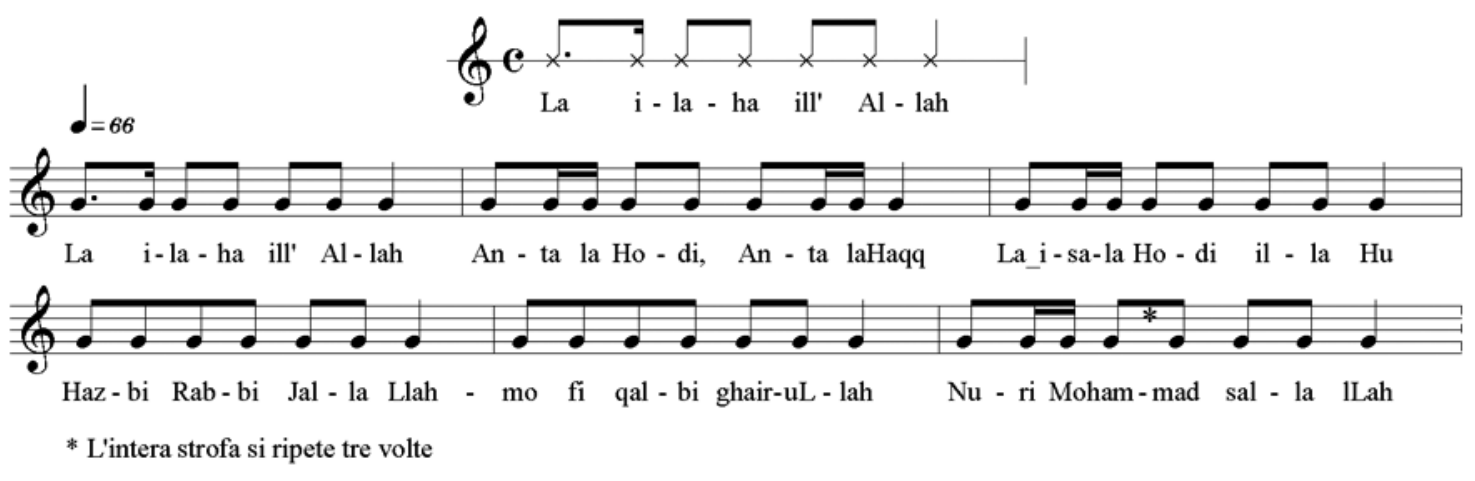

9 Nella trascrizione e traslitterazione che ne fa l'etnomusicologa uzbeca Razia Sultanova il testo dell'inno sacro, traslitterato secondo l'uzbeco corrente, risuona: Bismillahi-r-rahmoni-r-rahïm/Antalho dey antalhaqq, laysAllāh $\bar{u}$ illahū,/Asli rabbim Jallolloh, mohi qalbim xayrulloh/Nuri Muhammad sallalloh, lo ilaha illalloh (6 volte). Cfr. Razia Sultanova, Poyushčee slovo uzbekskikh obryadov (Opït liričeskogo issledovaniya), Yana, Tashkent, 1994: 104-105. 
Tra le comunità del Fergana, nel corso della pratica l'Antalho si contraeva divenendo l'attestazione di fede (shahāda): lā ilāha illā Llāh ("Non v'è altro dio se non Iddio").
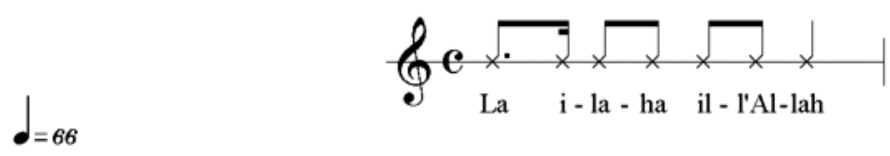

La shahāda si contraeva ulteriormente nella formula illā Llāh ("Se non Iddio") ripetuta su tempo sostenuto. Quando il tempo si era stabilito, poteva iniziare il canto di una composizione poetica di argomento devozionale/religioso che si incardinava sulla formula ritmica eseguita in ostinato.
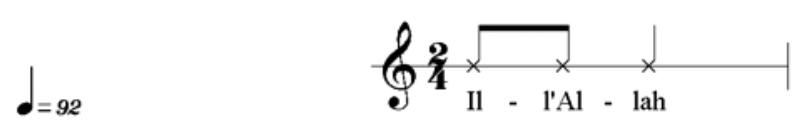

Lo zikr si contraeva quindi nella sola formula Allāh ("Iddio").
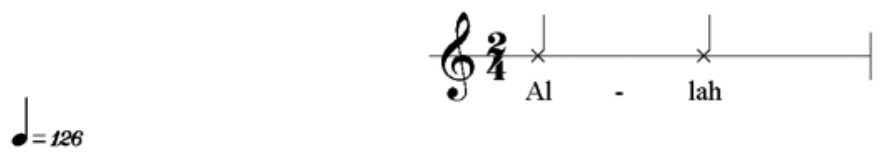

\section{ZIKR YEK ZARB}

Tra le comunità osservate, esiste un gruppo di zikr che vengono detti, usando un numerale, yek, do, se oppure chahār zarb ("zikr a un tempo, a due tempi, a tre tempi, a quattro tempi"). Il termine zarb, dall'arabo darb, significa letteralmente "colpo, battuta, tempo". Gli zikr yek, do, se e chahār zarb vengono fatti risalire ad 'Osman ricordato dai dervisci con il suo epiteto dhü-n-nürayn ("delle due luci"). ${ }^{10}$ Nello Zikr Yek zarb la formula è Allāh Hūwa, ("Iddio, Lui") in due tempi. Talora ascoltavo un accento forte: Allàh, che veniva spesso sostituito dal solo Ha!, oppure anche " $m m h$, $m m h$ " a bocca chiusa, che ricorda l'Ah-Hum pronunciato dai dervisci naqshband̄̄ âfāqu del vicino Xinjiang. Il cerchio dei dervisci ripeteva Hūwa in modo responsoriale. Allo zikr si abbinava un movimento evidente: il cerchio dello halqa, composto da dervisci seduti con le spalle che si toccavano e le mani sulle proprie ginocchia, inizia ad inchinarsi verso il centro così che le teste dei singoli sembrano convergere verso il centro del

10 'Uthmān ibn 'Affān (m. 655) fu il terzo califfo del neonato Islam. Egli viene ricordato qui come 'Osman dhü-n-nurayn, ossia "possessore delle due luci", perché avrebbe sposato due figlie del Profeta, Ruqayya (Rukiya) e, più tardi, 'Umm Kulthūm. Gli intervistati mi dicevano che a lui sono riconnessi gli zikr yek, do, se e chahār zarb. 
cerchio, secondo uno schema molto antico testimoniato da miniature e foto della fine del XIX.
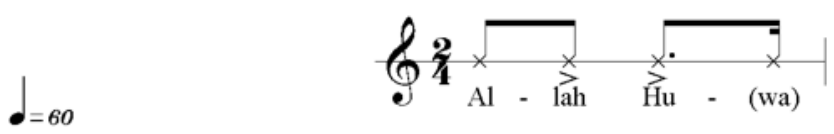

\section{ZIKR DO ZARB}

Allāh Hū, Hūwa, ("-Iddio-, Lui, Lui"). Da qui in avanti, nella trascrizione degli zikr do, se e chahār zarb inserirò sempre tra parentesi una ("a") indicando che può essere pronunciata o meno.
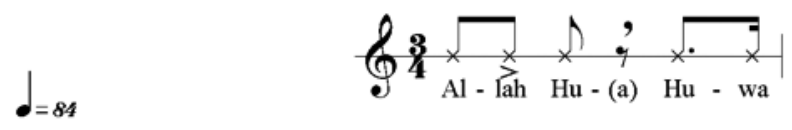

\section{ZIKR SE ZARB}

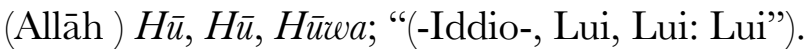
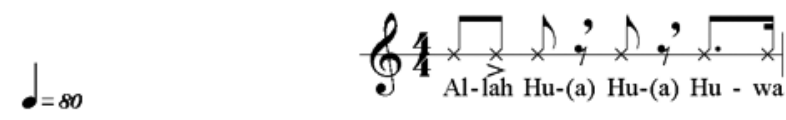

\section{ZIKR CHAH $\bar{A} R$ ZARB}

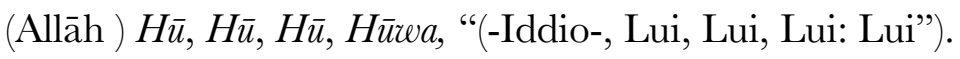
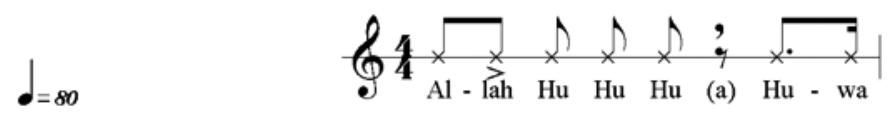

\section{ZIKR DI ZANGI BUWA (ZANGI ĀTA)}

Hayy Allāh Hūwa (Ah) Hū, Hū, Hūwa! (Oh) Hayy Allāh Hūwa (Ah) Hū, Hū, Hūwa. Questo particolare zikr deriverebbe da un episodio della vita del suf yasavī Zangī Āta (anche Zangi Ôta, Zangi Bāba e Zangi Buwa) il quale giunse da Tashkent sino a Safìd Bulān, nell'attuale Kyrgyzstan e vi fece ritorno, in un giorno solo. Il viaggio miracoloso si sarebbe compiuto in virtù di questo zikr che egli praticava lungo la via. 


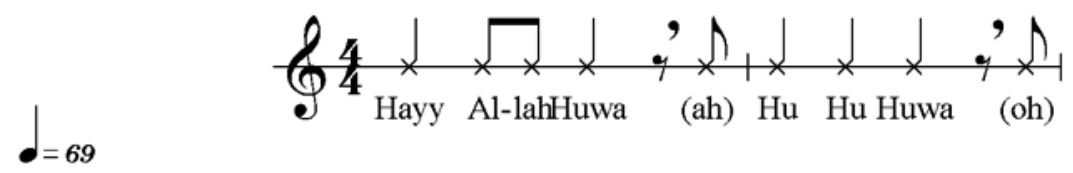

\section{ZIKR-I ARRA (ANGHE ARRA DAM, ARRA TORTADURLAR)}

Letteralmente "zikr della sega", "zikr del saracco", ma anche: "zikr del Profeta Zakariyya". La formula è: Allāh, Hūu Allāh Hū! ("Iddio, Lui! Iddio, Lui!"). Secondo gli intervistati, questo particolare zikr deriverebbe da un episodio della vita del Profeta Zaccaria che, inseguito da dei nemici, si sarebbe nascosto nel tronco di un albero cavo, confidando nell'albero e nella buona sorte invece che in Iddio. I nemici, invece, individuarono il suo nascondiglio e iniziarono a segare il tronco dell'albero e, dopo un colloquio con Dio, il Profeta iniziò questo particolare, intenso, zikr noto ad orientalisti ed etnomusicologi come prerogativa della confraternita Yasaviyya:
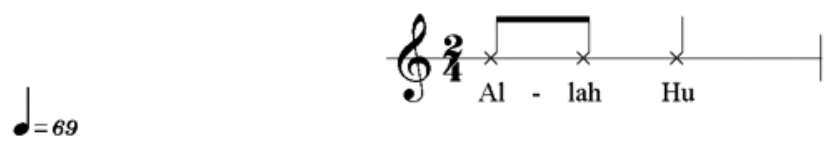

\section{ZIKR NA DOMAD}

("Zikr di colui che non tornò”) sulle sillabe: Ah, Hū! Ah, Hūu ("Ah, Lui! Ah, Lui! Ah, Lui"). Secondo gli intervistati, la genesi di questo zikr sarebbe legata ad Ahmad Yasavī: egli mandò il figlio alla tribù dei Suri a chiedere qualcosa ma questi lo uccisero, gli tagliarono la testa e la fecero portare ad Aḥmad Yasavī su di un piatto da frutta, come fosse un melone. Alla sua vista Yasavī avrebbe iniziato questo zikr. ${ }^{11}$
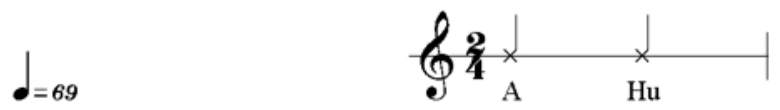

\section{SUL RAQS-I SAMĀ'}

Come si sa, il termine arabo samā' può essere reso con "ciò che viene udito, ciò che viene ascoltato" e, per estensione, "l'audizione; l'ascolto"; solo in seguito esso assunse il significato più tecnico e confrerico di "concerto spirituale". Storicamente le prime tracce di questa pratica datano alla metà del III secolo dell'Egira/X sec. d.C. e ci conducono tra i circoli sufi di Baghdad, capitale culturale dell'epoca, da dove il sam $\bar{a}^{`}$ si sarebbe progressivamente diffuso

11 Si noti il profondo collegamento tra un momento di estrema difficoltà, di pericolo, nella vita di un santo o di un Profeta, e la nascita di uno zikr, quasi che esso nascesse in condizioni di grande avversità. 
nelle aree vicine. I trattati dell'epoca, scritti soprattutto in difesa dai detrattori, mettono in primo piano il grado di raccoglimento e di ascolto che si è capaci, o meno, di attivare. Da questa messa in primo piano di un ascolto assoluto deriva un concetto radicalmente diverso di "musica", da ascoltarsi con un altro orecchio, come bene sintetizzerà Jalāl-ud Dīn Rūmī (1207-1273), e per altri fini che vanno di là dal piacere, pur partendo da esso.

Anche in area centroasiatica si ebbero numerosi trattati in accusa o in difesa della pratica, ma quel che più importa rilevare qui è che non sono giunti ai nostri giorni cerimoniali di sama $\bar{a}^{\varsigma}$, com'è invece accaduto altrove nel vasto mondo del sufismo (tasawrouf) ${ }^{12}$. Non so dire se questa tabula rasa dipenda dalla violenta repressione sovietica verso le attività del sufismo così come di qualsiasi altra cultura spirituale che viveva nell'area. Nel corso delle mie ricerche ho però potuto raccogliere la testimonianza di un derviscio anziano, M. Kh. di Kokand, che mi descrisse uno zikr che si faceva a Turkistan ${ }^{13}$ negli anni 1940-50, quand'era giovane, senza soldi, e doveva chiedere piangendo al controllore del treno di lasciarlo salire senza biglietto per recarsi a Turkistan: i dervisci si disponevano su due file parallele verso il mị̂rāb, non so dire se di una dimora privata o di una moschea, e intonavano La Haw, La haw, La haw accompagnandosi con la viella ad arco ghija $k^{14}$. Di fronte alla videocamera M. Kh. mi mostrava anche un accenno di zikr cantato, ritmato, abbinato a moti fisici: eretto, da fermo, M. Kh. ruotava il tronco a destra e a sinistra facendo perno sulla spina dorsale, e le braccia si muovevano di conseguenza: vedendole dall'alto, esse formavano una S. La testimonianza di M. Kh. mi fa immaginare una realtà differente nella quale $d h i k r$ e $s a m \bar{a}^{`}$ interagivano, punteggiati da moti fisici e da strumenti musicali.

Di là dalle rêveries dell'autore, per capire meglio la particolare posizione di Adil Khān e della sua via, va detto che i naqshband, tranne rare eccezioni, non accolsero il $\operatorname{sam}_{\bar{a}}{ }^{\natural}$ tra le loro pratiche. Il motivo di questa scelta mi sembra bene espresso da due massime dei maestri: 'Abd al-Khāliq Ghujudwānī (m. 1220), consiglia al suo successore, Khwāja Awliyā' Kabīr: "Non impegnarti troppo nella musica e nella danza sacra (nell'originale persiano, $s a m \bar{a}$ ), poiché indulgervi è pericoloso per la vita del cuore. Ma non rigettare la danza sacra $\left(s a m \bar{a}{ }^{\natural}\right)$ poiché

12 Si pensi solo alla tradizione ottomano-turca dei dervisci mevlevi, più noti in Occidente come "dervisci rotanti", oppure agli specifici repertori nati nella Chistiyya indopakistana che rientrano nel genere qawrwali.

13 Turkistan è oggi un centro dell'attuale Kazakhstan meridionale; anticamente si chiamava Yasi ed è qui che visse e fu sepolto Aḥmad Yasavī, facendo divenire Yasi la casa madre dei dervisci yasavī d'Asia Centrale. Tamerlano (Timūr-i Lenk) nel XV secolo fece erigere un maestoso mausoleo, visitabile ancor oggi, in memoria del grande maestro.

14 La frase cantata dai dervisci potrebbe essere riconducibile alla nota formula: lā hawlā wa lā quwwatā illa bi Llāh ("Non vi sono forza e potenza se non in Iddio"). La viella ad arco ghijāk è una viella ad arco della famiglia "a puntale". 
molti vi fanno affidamento." ${ }^{15}$. Un’identica posizione sarà espressa più tardi da un elegante ed eloquente gioco di parole di Bahā ud-Dīn Naqshband (m. 1389), il quale sostenne: "Noi non lo pratichiamo e non lo condanniamo." (mā na ìn kār mīkonìm, na inkār mīkonīm) ${ }^{16}$.

Il caso dei naqshband̄̄ jahrī incontrati nel Fergana rientra tra le eccezioni di cui sopra, e mi sembra possa essere ricollegato ad una tradizione naqshband̄ che sorse tra Samarkand, Kashgar e le sei città oasi (altıshahr) poste nel bacino del Tarim, sui bordi del deserto del Taklamakan, nei territori dell'attuale Xinjiang cinese. Questa mia ipotesi nasce da alcuni dati: innanzitutto la comune area geo-culturale, poiché la valle, divisa dopo il 1924 tra i confini di Uzbekistan, Tajikistan, Kazakhstan e Kyrgyzstan, fu costantemente in contatto con la Cina. Nel corso dei millenni essa fece parte di macro-aree dette con termini diversi, quali Saka, Tocharistan, khanato di Chagatay o Turkestan orientale. L'ultima di esse, prima dell'invasione russa e dell'amministrazione sovietica, fu quella del khanato di Kokand (17101876), la cui propaggine orientale era Kashgar, nell'attuale Xinjiang. Naturalmente i dervisci, soprattutto quelli vaganti detti qalandar, si muovevano incuranti di potentati e confini, bensì in una loro rete autonoma costellata dalle dimore dei maestri. In questo senso, come avrei appreso più tardi, la genealogia (silsila, shajara) di discendenza spirituale di Adil Khān consultata da Zarcone (2006: 46) ci dice che egli fu allievo di Sayyid Supikhan Ishān (m. 1973), discendente di Khwāja Buzurg Khān di Kashgar. Questo spiegherebbe la vicinanza tra il rituale osservato ad Andijān e altri osservabili tra gli uiguri della naqsbandiyya āfāqiyya, sui quali mi tratterrò nel prossimo paragrafo.

Ignaro di tutto questo, nella tarda sera del 22 maggio 2003, superando pozzanghere e bambini che giocavano, arrivai con l'etnografo Adham Ashirov nel quartiere (mahalla) di case in argilla disseccata dov'era la dimora di Adil Khān. Lo zikr era già in corso e si udiva sin dal vicolo ${ }^{17}$. Alcuni devoti controllavano la porta di ingresso; fummo introdotti di soppiatto ed entrammo velocemente, cercando di non dare nell'occhio. Giunti nel cortile pergolato chiesi allo stesso Adil Khān, con un gesto, il permesso di registrare e lui acconsentì. All'interno, un affollato circolo maschile di zākir seduti a terra su dei korpache in cotone imbottito. Adil Khān, rientrato, era parte del circolo e sedeva dando la schiena al mihrāab. Le età erano diverse e c'erano anche

15 Mawlana Ali ibn Husain al-Waiz Kashifi Safi, Rashahat Ain al-Hayat. Beads of Dew (from the Source of Life). Histories of the Khwajagan, the Masters of Wisdom, traduzione e cura di Muhtar Holland, Al Baz Publishing, Fort Lauderdale, 2001: 16.

16 La massima di Bahā ud-Dīn Naqshband proviene dalle Nafahăt al-Uns ("Le vite dei santi") di 'Abd ar-Rahmān Jāmī (1414-1492). Cfr. Demetrio Giordani, I naqshbandi. Uomini storia e dottrine di un ordine sufi, Jouvence, Sesto San Giovanni (MI), 2019: 67.

17 Come scrivevo in una nota precedente, il volume sonoro dello zikr jahrī è stato uno degli elementi che lo ha fatto riconoscere, identificare e proibire dalla polizia sovietica, laddove lo zikr khā $f \bar{\imath}$, praticato silenziosamente, ha potuto sopravvivere meglio alla represseione. 
dei bambini. Sull'ostinato dello zikr se zarb descritto più sopra, alcuni zākir si levavano in piedi e si disponevano sui quattro punti cardinali di un circolo più piccolo, interno a quello formato dagli zākir seduti a terra. Da ogni punto cardinale i quattro si dirigevano con un ampio passo verso il centro del cerchio costituito dai loro corpi e, a ritmo, univano le mani in alto, convergendo su di un centro posto sopra le loro teste, con un gesto della mano simile al "fare canestro" nel gioco del basket. Contemporaneamente essi rotavano intorno al centro del cerchio da loro creato, come nell'arte marziale cinese Ba Gua. Altri si aggiungevano a questo moto. ${ }^{18}$ Complessivamente, in questo incontro cerimoniale detto raqs-i sam $\bar{a}^{\varsigma}$ (più raramente jāmlānish) si sommavano lo zikr se zarb, i raqs dei dervisci dal perimetro al centro del cerchio, che progressivamente rotava, e il canto di un poema spirituale da parte di un qāa'. Ad un segnale dello halqa bash ("capo del circolo") questa fase si concludeva e i presenti ritornavano a sedersi in circolo. Per poi passare ad altre fasi dello zikr. Nella mia pochezza percepivo comunque una grande energia controllata e notavo pianti e stati alterati tra i presenti.

\section{IL POSSIBILE RETROTERRA GULTURALE DEL RAQS-I SAMĀ‘ DI ANDIJĀN}

I dibattiti tra jahriyya e khāfyyya giunsero talora a dei punti di intesa: un esempio è dato dagli scritti del noto sheykh naqshband̄̄ Aḥmad ben Maulana Jalāl ad-dīn al-Kasān̄̄ ad-Dahbīdī (Samarkand, 1461-1542), più noto con lo pseudonimo di Mahdum-i A'zam ${ }^{19}$. In particolare, l'opera di Dahbīdī costituì il fondamento dottrinale per un suo diretto successore come fu Ishāq Khwāja, il quale verso il 1580 si mosse da Samarcanda per raggiungere l'area delle sei città-oasi dette altıshahr("le sei città"), ossia Kashgar, Yarkand, Khotan, Turfan, Aqsu e Kucha) poste sui bordi del deserto del Takla Makan. Giunto con i suoi compagni nell'area delle altıshahr egli diede vita ad un ramo detto naqshband̄̄yya-ishāqiyya. Qui Khwāja Ishāq iniziava la particolarissima pratica dei samā' pubblici ai quali partecipavano centinaia di persone definendoli raqs $\bar{u} \operatorname{sam}^{\mathfrak{c} 20}$. Soprattutto, Khwaja Ishāq mise in relazione il $\operatorname{sam}^{\varsigma}$ con la forza

18 Una descrizione del cerimoniale anche in Thierry Zarcone, "Les danses naqshbandī en Asie Centrale et au Xinjang: Histoire et actualité" in fournal d'Histoire du Soufisme, n. 4, Maisonneuve, Paris, 2004: 185.

19 Su di lui e su alcuni suoi scritti cfr. Alexandre Papas, "No Sufism without Sufi Orders: Rethinking Tarīqa and Adab with Aḥmad Kāsānī Dahbidī (1461-1542)", Kyoto Bulletin of Islamic Studies, Kyoto, Center for Islamic Area Studies at Kyoto University (KIAS), 2-1, 2008: 4-22. Nella prospettiva delle prossime righe si segnala in particolare il suo Risala-yi sama iya, ("Epistola sul samā"'), Ms. MNS IVANUz (Institut Vostokovedenie Akademii Nauk Uzbekistan), N 501/11, ff. 13 $3^{\text {b-3b. }}$

20 Cfr. Alexandre Papas, “'Dansez et chantez': le droit au samā'selon Āfāq Khwaja, maītre naqshbandi du Turkestan (XVII)", in Fournal d'Histoire du Soufisme, n. 4, Maisonneuve, Paris, 2004: 171. Secondo l'indagine di Papas, il termine raqs $\bar{u}$ sam $\bar{a}^{\varsigma}$ compare nel manoscritto, di natura agiografica su Khwāja Ishāq: cfr. Ms Tadhkira-yi Natā'-̄j al-arifīn, KBJT (Kitobkhonaji Buzurgi Jumhurii Tojikiston), 1802: 190. 
detta jadhba ("attrazione divina, rapimento in Iddio"), che rapisce e attira potentemente a sé il praticante, e diede vita ad un inedito jadhba dar-sam $\bar{a}^{6}$ ("rapimento nel sama ${ }^{\text {'" }}$ ). Secondo la sua concezione, la forza del rapimento poteva agire in maniera "improvvisa e immediata" ${ }^{21}$ sulle centinaia di dervisci o di semplici fedeli che partecipavano al sama $\bar{a}^{`}$, come avvenne ad esempio ad Āqsū quando: "ogni uomo di Āqsū giunse all'attrazione divina e si fece immediatamente discepolo e fedele"22. Una simile "attrazione" delle centinaia di presenti avveniva però in virtù della concentrazione (tawajjuh) e della forza catalizzatrice dei maestri naqshband̄̄ che presiedevano ai cerimoniali ${ }^{23}$. Khwaja Ishāq fece ritorno a Samarcanda per lasciare questo mondo nel 1599.

In questo particolarissimo contesto giunse Āfāq Khwāja (? -1693/94), che fu insieme derviscio naqshbandī e re del Turkestan orientale dal 1681 al 1694. La sede del suo regno fu Kashgar, allora centro culturale di primissima grandezza sulla rete delle altıshahr. Durante il suo regno Āfāq Khwāja intensificò le pratiche istituite da Khwāja Ishāq, promuovendo e incrementando pratiche pubbliche di $s a m \bar{a}^{\varsigma}$, tratto davvero inedito per un cerimoniale da sempre piuttosto "appartato" e riservato a pochi: in questo senso il Turkestan orientale costituisce un esempio piuttosto raro di "sufismo di stato"24.

Gli zikr jahrī del ramo naqshbandī âfāq $\bar{\imath}$, molto particolari per il loro numero e per i loro gradi di complessità formale ${ }^{25}$, sopravvivono ancor oggi, di nascosto dalle autorità cinesi, e si possono ascoltare in alcune registrazioni effettuate da Jean During nel 1988 nell'attuale Xinjiang ${ }^{26}$. Più di recente ho potuto visionare intensi cerimoniali sufi, non solo naqshband $\bar{\imath}$, filmati nello Xinjiang dal giovane $\mathrm{Mu}$ Qian per la sua tesi di dottorato inedita ${ }^{27}$.

21 Ancora Ms Tadhkira-yi Natā'-ìj al-arifìn, 1802: 83 in Papas 2004: 172.

22 Papas traduce dal manoscritto Anīs al-tālibīn, Ms Ind. Inst. Pers. 45 folio 90b.Cfr. Alexandre Papas Soufisme et politique entre Chine, Tibet et Turkestan, Paris, Jean Maisonneuve, 2005: 54-55.

23 Papas, 2005: 55.

24 Approfondisce la questione dei rapporti tra spiritualità e politica Papas, 2005.

25 Cfr. Mukaddas, Mijit, À la recherche de la voie des anciens. Les chants soufi de la Naqshbandiyya de Ghulja chez les Ouïgours, Master II, Université de Paris-Sorbonne, musicologie, Ethnomusicologie, 2008 (tesi di dottorato, non pubblicata).

26 Un loro estratto è stato pubblicato in: "Zikr Naqshbandi of Turkestan", in Trance 2, Musical Expeditions, Ellipsis Arts, Roslyn, N.Y., 1995, n 4010. Quindi nel doppio CD: The Silk Road: a musical caravan, Smithsonian Folkways Recordings, Washington, 2002. CD: 40438. Di recente un nuovo estratto, forse più accessibile al lettore italiano, si trova nella traccia 11 del CD allegato a Jean During, Musica ed Estasi. L'ascolto mistico nella tradizione suf, traduzione e cura di Giovanni De Zorzi, Roma, SquiLibri, 2013.

$27 \mathrm{Mu}$ Qian, Experiencing God in Sound: Music and Meaning in Uyghur Sufism, University of London, School of Oriental and African Studies (SOAS), 2019, PhD Thesis (non pubblicata). 


\section{NOTE SUI METODI}

Lasciando la parola ai dervisci incontrati, va innanzitutto notata una caratteristica del metodo jahrī da loro spesso sottolineata: solo lo jahrī può farsi strada nel mondo fenomenico arrivando a toccare l'animo dell'ascoltatore, investito, volente o nolente, dalla sua onda sonora. Come direbbero gli antichi trattatisti, lo jahrì è capace di agire come un richiamo ad alta voce che risveglia un dormiente dal suo torpore. Questo implica un suo potere terapeutico: lo zikr jahri "fa del bene" anche a coloro che lo ascoltano, toccandoli intimamente, risvegliandoli dalla distrazione (ghaflat) nella quale conducono le loro vite, proprio come farebbe un forte richiamo sonoro ad un dormiente. In questa sua "sonorità" è immanente una dimensione estetica: lo zikrjahrī si connota per il trasporto, il piacere, la gioia e per il senso di bellezza che provoca non solo in chi lo pratica, ma anche in chi lo ascolta di lontano. Questa dimensione estetica compare nei trattatisti del passato così come nelle interviste realizzate durante la mia ricerca.

Come mi ricordava la studiosa Ibodat Rajabova, intervistata a Bukhara, la dimensione sonora dello zikr jahrī richiede però che si sia "fuori dal mondo" (tark-i dünya) per poterlo praticare, mentre lo zikr khăfì può essere praticato anonimamente in ogni momento della vita. E, come si è visto più sopra, la meravigliosa sonorità dello zikr jahrī ha fatto anche sì che il suo suono giungesse sino alla polizia, con risvolti negativi in epoca russo-sovietica.

Ancora secondo gli intervistati, nello zikr jahri lavorano insieme $q \bar{a} l b$ ("cuore") jazat, o anche tana ("corpo") e til ("lingua"), mentre nello zikr khāfì, invece, si lavora con il solo qälb. Il sudore e le lacrime che fuoriescono dallo zākir nel corso dello zikr jahri bruciano e rimuovono le proprie occlusioni spirituali e, in qualche modo, si riconnettono allo stesso Profeta: secondo l'intervistato Hajji A. M., infatti: "Quando Muhammad fece zikr dal suo corpo uscì acqua; dall'acqua uscita Allāh creò la Luna e il Sole, l'arsh, ("trono"), il kursī ("sgabello"), il qalam mahfüz ("il calamo o la scienza divina"): tutti gli esseri furono creati dall'acqua che uscì dal corpo di Muhammad."

Il suono raschiante e fragoroso dello zikr-i arra viene paragonato sin dal nome ad una sega o ad un saracco oppure ad una raspa: esso possederebbe la capacità di trasformare le condizioni psichiche dello zākir e tra esse, ovviamente, le "emozioni negative". Secondo gli intervistati questa pratica sarebbe stata trasmessa dall'enigmatico Khidr (Khezr) a Khoja Aḥmad Yasavī, proprio allo scopo di allontanare da sé la sua condizione di sconforto.

Piace concludere ricordando che lo zikr (jahri o khâầ che sia) provoca negli zākir l'intima consapevolezza di un rapporto ravvicinato con l'Altissimo, secondo il noto versetto coranico: 
Fa-zkurūnī azkurkum, "Ricordatevi dunque di Me e io mi ricorderò di voi"”. va ricordata la tradizione secondo la quale un cerchio (halqa) d'angeli si forma al di sopra del cerchio degli umani intenti a ricordare Iddio. In una delle molte versioni, narrata dal Profeta stesso e riportata da Abu Hurayra:

Il Profeta disse: 'Iddio ha degli Angeli eminenti che viaggiano di continuo a seguire attentamente le sedute di $z i k r$, e quando trovano un consesso nel quale si fa $z i k r$ siedono con loro e si circondano l'un l'altro colle loro ali fino a riempire lo spazio tra loro e il cielo del mondo; e quando se ne allontanano salgono sino ad arrivare fino al cielo, e Iddio Potente e Glorioso li interroga -ed Egli ne sa di più-: 'Da dove venite?'; 'Veniamo da presso i Tuoi servi sulla terra -rispondono- che Ti esaltano, Ti magnificano, proclamano la Tua Unicità, Ti lodano e Ti invocano. ${ }^{29}$

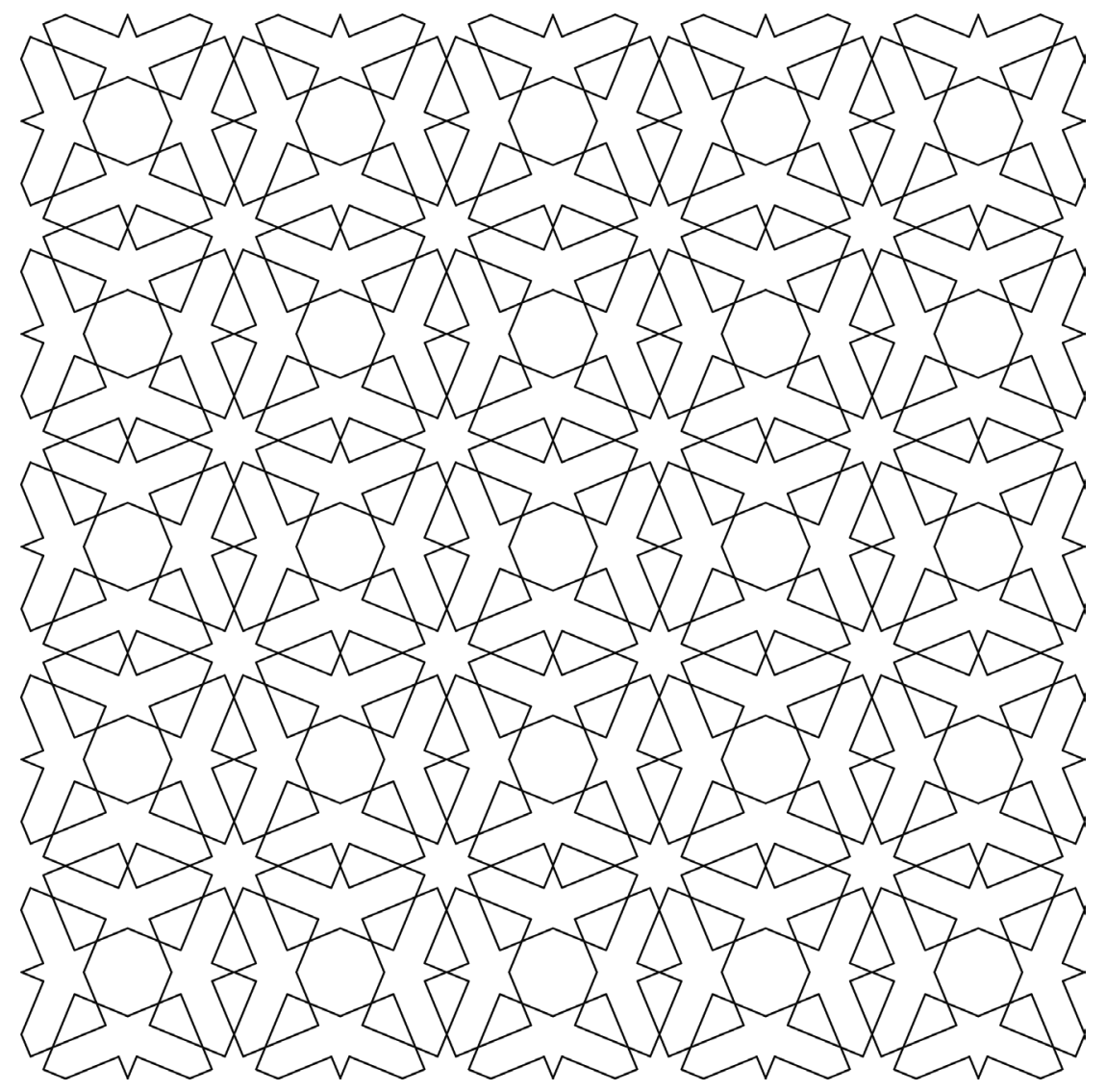

28 Corano, Surat al-Baqara, II: 152.

29 Hadith ("detto") del Profeta riportato da Abu Hurayra e presente in Muhyī ad-Dīn an-Nawawī, Il giardino dei devoti (Ryadh as-Salihīn). Detti e fatti del Profeta, traduzione e cura di Angelo Scarabel, Società Italiana di Testi Islamici, Trieste, 1990: 390. 


\section{BIBLIOGRAFIA DI RIFERIMENTO}

ALGAR, Hamid, "A brief history of the Naqshbandī Order" in Marc GABORIEAU, Alexandre POPOVIC e Thierry ZARCONE (a cura di), Naqshbandis. Cheminements et situation actuelle d'un ordre mystique musulman, Işin, Istanbul, 1990: 3-44.

BABADJANOV, Bakhtiyar, 2002, "Une nouvelle Source sur les rituels de la tarīqa Yasaviyya. Le risala-i dhikr-i sultān al-'arifīn", in Gournal d'Histoire du Soufisme, n. 3, Maisonneuve, Paris: 223-228.

BABADJANOV, Bakhtiyar - GHÜLAMOV, Sanjar, "Ritual practice of Sufi communities in Mavara'annahr (18th-19th centuries)" in Central Asia on Display. Proceedings of the VII European Society for Central Asian Studies Conference, a cura di Gabriele Rasuly-Paleczek e Julia Katschnig, LIT Verlag, Wien, 2004, Volume 1: 297-308.

BENNINGSEN, Alexandre - REMERCIER-QUELQUEJAY, Chantal, L'Islam parallelo. Le confraternite musulmane in Unione Sovietica, Marietti, Genova, 1990. (ed. or. Le soufi et le commissaire, Editions du Seuil, Paris, 1986).

Il CORANO, Introduzione, traduzione e commento di Alessandro Bausani, Rizzoli, Milano, 1988.

DE ZORZI, Giovanni,

2006, Gli zikr della confraternita sufi Yasaviyya nella valle del Fergana (Uzbekistan, Kazakhstan, Kirgyzstan). Relatore Francesco Giannattasio; correlatore Giovanni Giuriati. Tesi di dottorato di ricerca in Storia e Analisi delle Culture musicali, XVII ciclo, Dipartimento di studi Glottoantropologici e musicali dell'Università "La Sapienza” di Roma (non pubblicata).

2008, "Incontri di dervisci a Karkhidon. Lo zikr ("menzione, ricordo") dei Nomi Divini in area centroasiatica" in Phoenix. In domo Foscari, numero 1, anno 2008, Scriptaweb, Napoli: 89-128.

2013, “The Jahrī Concept in Central Asia and its Implications in Sufism, Music and Therapy". https://www.akdn.org/akmi/musical-geographies-central-asia/giovanni-de-zorzi (Atti online del convegno "Musical Geographies of Central Asia", organizzato da Middle East and Central Asia Music Forum; Institute of Musical Research-School of Advanced Study; School of Oriental and African Studies (SOAS)-University of London; Aga Khan Music Initiative-Aga Khan Trust for Culture, Londra, 18-19 maggio 2012). Pagina web visitata il 25.01.2021

2013a, "Con dervisci naqshbandī jahrī nella valle del Fergana" in Con i dervisci. Otto incontri sul campo, a cura di Giovanni De Zorzi, Milano, Mimesis, 2013: 173-210.

DURING, Jean, 2013, Musica ed Estasi. L'ascolto mistico nella tradizione sufi. Traduzione e cura 
di Giovanni De Zorzi, Roma, SquiLibri. (ed. or. Musique et Extase. L'audition mystique dans la tradition soufie, Paris, Albin Michel, 1988).

FATHI, Habiba, 1998, "Le pouvoir des otin, institutrices coraniques, dans l'Ouzbékistan indépendant", in Boukhara la noble. Cahiers d'Asie Centrale, 5-6, Édisud, Tachkent-Aix en Provence: 313-331.

GARRONE, Patrick, Chamanisme et Islam en Asie Centrale, Maisonneuve, Paris, 2000.

GIORDANI, Demetrio, I naqshbandi. Uomini storia e dottrine di un ordine sufi, Jouvence, Sesto San Giovanni (MI), 2019.

LEVIN, Theodore, The hundred thousand Fools of God. Musical travels in Central Asia (and Queens, New York), Indiana University Press, Bloomington, 1996.

MIJIT, Mukaddas, À la recherche de la voie des anciens. Les chants soufi de la Naqshbandiyya de Ghulja chez les Oügours, Master II, Université de Paris-Sorbonne, musicologie, ethnomusicologie, 2008 (n. p.).

an-NAWAWĪ, Muhyī ad-Dīn, Il giardino dei devoti (Riyadh as-Salihīn). Detti e fatti del Profeta, traduzione e cura di Angelo Scarabel, Società Italiana di Testi Islamici, Trieste, 1990.

NEUBAUER, Ekhard - DOUBLEDAY, Veronica, 2001/2002, "Islamic Religious Music. 3 Dhikr and sufi ritual" in The New Grove Dictionary of Music and Musicians, Macmillan Publishers, Volume 12.

PAPAS, Alexandre,

2004, "'Dansez et chantez': le droit au samā' selon Āfāq Khwaja, maītre naqshbandi du Turkestan (XVII)", in Fournal d'Histoire du Soufisme, n. 4, Maisonneuve, Paris, 2004: 169-180.

2008, "No Sufism without Sufi Orders: Rethinking Tarīqa and Adab with Aḥmad Kāsānī Dahbidī (1461-1542)", Kyoto Bulletin of Islamic Studies, Kyoto, Center for Islamic Area Studies at Kyoto University (KIAS), 2-1: 4-22.

2010, Mystiques et vagabonds en Islam: portraits de trois soufis qalandar, Paris, Éditions du Cerf, coll. "Patrimoines - Visages de l'Islam".

QIAN, Mu, Experiencing God in Sound: Music and Meaning in Uyghur Sufism, University of London, School of Oriental and African Studies (SOAS), 2019, PhD Thesis (non pubblicata).

SAFI, Mawlana Ali ibn Husain al-Waiz Kashifi, Rashahat 'Ain al-Hayat. Beads of Dew (from the Source of Life). Histories of the Khwajagan, the Masters of Wisdom, traduzione e cura di Muhtar Holland, Al Baz Publishing, Fort Lauderdale, 2001.

SULTANOVA, Razia,

1994, Poyushčee slovo uzbekskikh obryadov (Opüt liričeskogo issledovaniya), Yana, Tashkent. 
2005, "En soulevant le voile: les chants des femmes dans les cultures traditionnelles turcophones", in "Cahiers des Musiques Traditionnelles", Genève, 2005, n.18: 11-38.

VUILLEMENOT, Anne-Marie,

"Quand un bakhshı kazakh invoque Allāh" in "Journal d'Histoire du Soufisme", n. 4, Maisonneuve, Paris, 2004: 131-141.

ZARCONE, Thierry,

2004, "Les danses naqshbandī en Asie Centrale et au Xinjang: Histoire et actualité" in fournal d'Histoire du Soufisme, n. 4, Maisonneuve, Paris, 2004: 181-198.

2006, "Bridging the Gap between pre-Soviet and post-Soviet Sufism in Ferghana Valley (Uzbekistan): The Naqshbandi Order between Tradition and Innovation" in Popular Movements and Democratization in the Islamic World, edited by Masatoshi Kisaichi, New Horizons in Islamic Studies, New York, Routledge, 2006: 43-56.

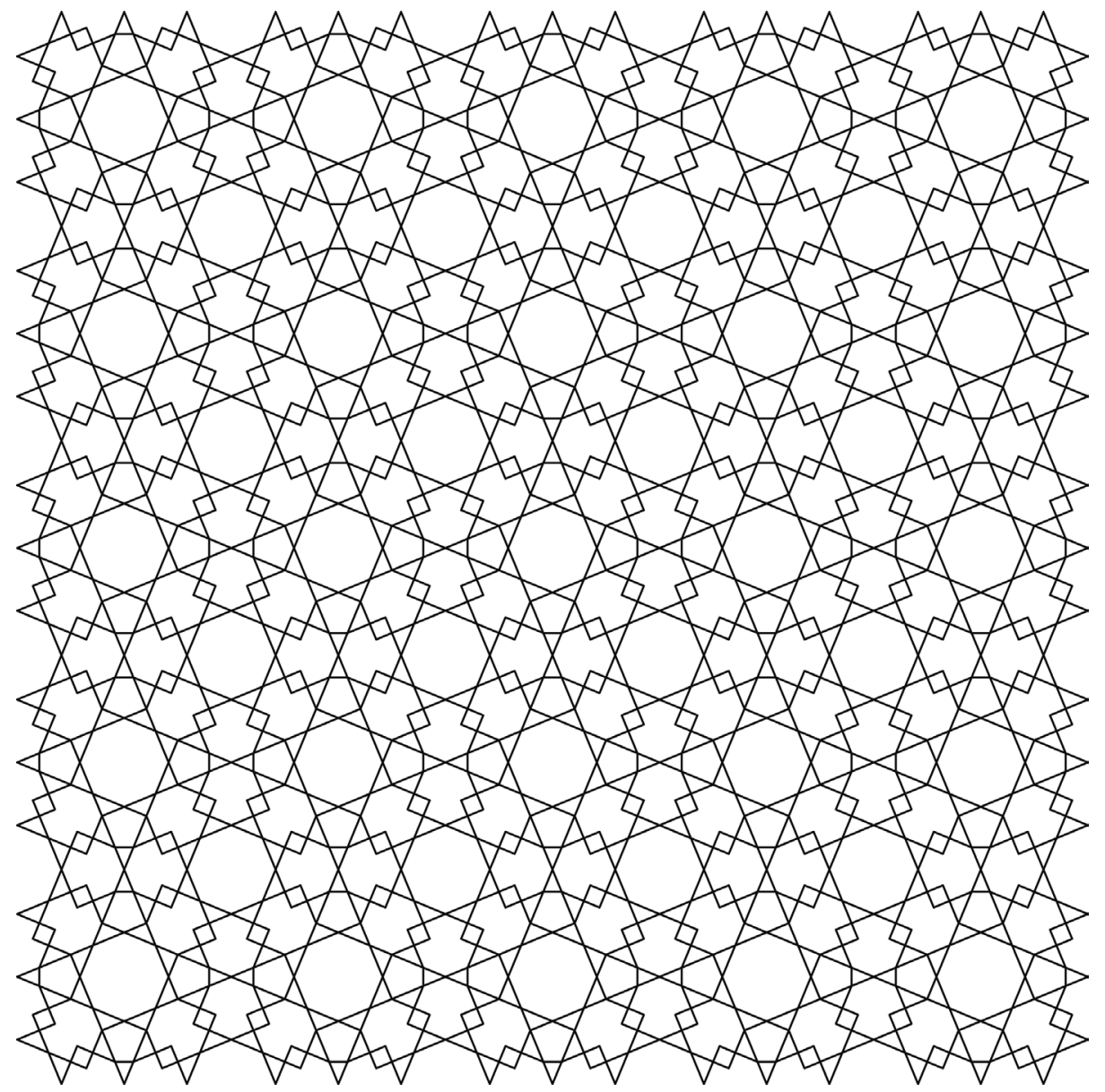

